\title{
Technological implementation of a photonic Bier-Glas cavity
}

Jurkat, Jonathan; Moczala-Dusanowska, Magdalena; Jacobsen, Martin Arentoft; Predojevic, Ana; Huber, Tobias; Gregersen, Niels; Hoefling, Sven; Schneider, Christian

Published in:

Physical Review Materials

Link to article, DOI:

10.1103/PhysRevMaterials.5.064603

Publication date:

2021

Document Version

Peer reviewed version

Link back to DTU Orbit

Citation (APA):

Jurkat, J., Moczala-Dusanowska, M., Jacobsen, M. A., Predojevic, A., Huber, T., Gregersen, N., Hoefling, S., \& Schneider, C. (2021). Technological implementation of a photonic Bier-Glas cavity. Physical Review Materials, 5(6), [064603 ]. https://doi.org/10.1103/PhysRevMaterials.5.064603

\section{General rights}

Copyright and moral rights for the publications made accessible in the public portal are retained by the authors and/or other copyright owners and it is a condition of accessing publications that users recognise and abide by the legal requirements associated with these rights.

- Users may download and print one copy of any publication from the public portal for the purpose of private study or research.

- You may not further distribute the material or use it for any profit-making activity or commercial gain

- You may freely distribute the URL identifying the publication in the public portal 


\title{
Technological implementation of a photonic Bier-Glas cavity
}

\author{
Jonathan Jurkat $\odot,{ }^{1,}{ }^{*}$ Magdalena Moczała-Dusanowska, ${ }^{1}$ Martin Arentoft Jacobsen, ${ }^{2}$ Ana Predojević, ${ }^{3}$ Tobias Huber $\odot,{ }^{1}$ \\ Niels Gregersen ${ }^{\circ},{ }^{2}$ Sven Höfling $\odot{ }^{1},{ }^{1}$ and Christian Schneider ${ }^{1,4}$ \\ ${ }^{1}$ Technische Physik and Wilhelm Conrad Röntgen Research Center for Complex Material Systems, Physikalisches Institut, \\ Würzburg University, Am Hubland, Würzburg, Germany \\ ${ }^{2}$ DTU Fotonik, Department of Photonics Engineering, Technical University of Denmark, Ørsteds Plads 343, 2800Kongens Lyngby, Denmark \\ ${ }^{3}$ Department of Physics, Stockholm University, 10691 Stockholm, Sweden \\ ${ }^{4}$ Institute of Physics, University of Oldenburg, 26129 Oldenburg, Germany
}

(Received 23 September 2020; accepted 4 May 2021; published xxxxxxxxxx)

\begin{abstract}
In this paper, we introduce a quantum photonic device, which we term the photonic Bier-Glas cavity. We discuss its fabrication and functionality, which is based on the coupling of integrated $\operatorname{In}(\mathrm{G}(\mathrm{a})$ As quantum dots to a broadband photonic cavity resonance. By design, the device architecture uniquely combines the Purcell enhancement of a photonic micropillar structure with a broadband photonic mode shaping of a vertical, tapered waveguide, making it an interesting candidate to enable the efficient extraction of entangled photon pairs. We detail the epitaxial growth of the heterostructure as well as the necessary lithography steps to approach a GaAsbased photonic device with a height exceeding $15 \mu \mathrm{m}$, supported on a pedestal that can be as thin as $20 \mathrm{~nm}$. We further describe its key performance parameters using a Fourier-modal method. Finally, we present an optical characterization, which confirms the presence of broadband optical resonances, in conjunction with amplified spontaneous emission of single photons.
\end{abstract}

DOI: 10.1103/PhysRevMaterials.00.004600

\section{INTRODUCTION}

The preparation of single photons and entangled photon pairs is a critical resource in the fields of quantum optics, quantum metrology, and quantum information [1-3]. Quantum dots (QDs) embedded in microcavities are a promising candidate to create such nonclassical light states. The spontaneous emission enhancement experienced by the QD in a cavity is a valuable tool to reach very high photon coupling efficiencies into resonating modes and to boost the overall device efficiencies (the photon extraction and collection efficiency) beyond 75\% [4-6]. Furthermore, the spontaneous emission enhancement is key to mitigate the effects of pure dephasing on the quantum emitter by controlling the radiative transition lifetime. This enables the generation of highly indistinguishable photons, without the need for strong spectral filtering, which would decrease the system efficiency [7-9]. However, since in most implementations of coupled QDcavity systems, the mode volumes are in the order of $(\lambda / n)^{3}$, relatively high $\mathrm{Q}$ factors $\left(10^{3}\right.$ or higher $)$ are needed to facilitate a notable spontaneous emission enhancement. However, under those conditions, the Purcell effect becomes prominent within a small bandwidth only, which is prohibitive for the efficient extraction of entangled photon pairs. Broadband approaches based on photonic waveguides have been introduced to implement efficient single photon sources [10] and photon pair sources with improved characteristics $[11,12]$ based on III/V quantum dots. However, thus far, it turned out utmost

${ }^{*}$ Corresponding author: jonathan.jurkat@physik.uni-wuerzburg.de challenging, both from the modeling as well as the technology development, to combine the broadband performance of a photonic waveguide with the spontaneous emission enhancement of a microcavity.

Here, we address this problem, following a modification of a device suggestion proposed by Gregersen et al. [13]. By integrating a distributed Bragg-reflector (DBR) in a GaAsbased photonic trumpet, it was suggested to combine a modest quality factor cavity, supporting Purcell factors of up to 3, with the photonic waveguide effect that yields suppressed emission into leaky modes. While the initial suggestions considered a metallic back mirror [13], we implement a second DBR to mimic the symmetry of a DBR based micropillar. This also brings the advantage of a fully epitaxial structure, without the necessity of complicated wafer-bonding steps.

Our redesigned device resembles the shape of a German Bier-Glas. This shape consists of a taper section which contains the waveguide and the DBRs and a foot that shows an inverted taper. A device of this shape theoretically supports an extraction efficiency up to 0.725 together with a Purcell factor up to 3 . We demonstrate the modeling as well as the necessary technology for fabrication of these seemingly fragile object and our optical characterization verifies the presence of optical resonances, as well as pronounced, bright QD emission signals.

\section{MODELING}

We first perform a numerical investigation of the performance of the Bier-Glas geometry. A sketch of a simplified device geometry is depicted in Fig. 1(a). For a wavelength 


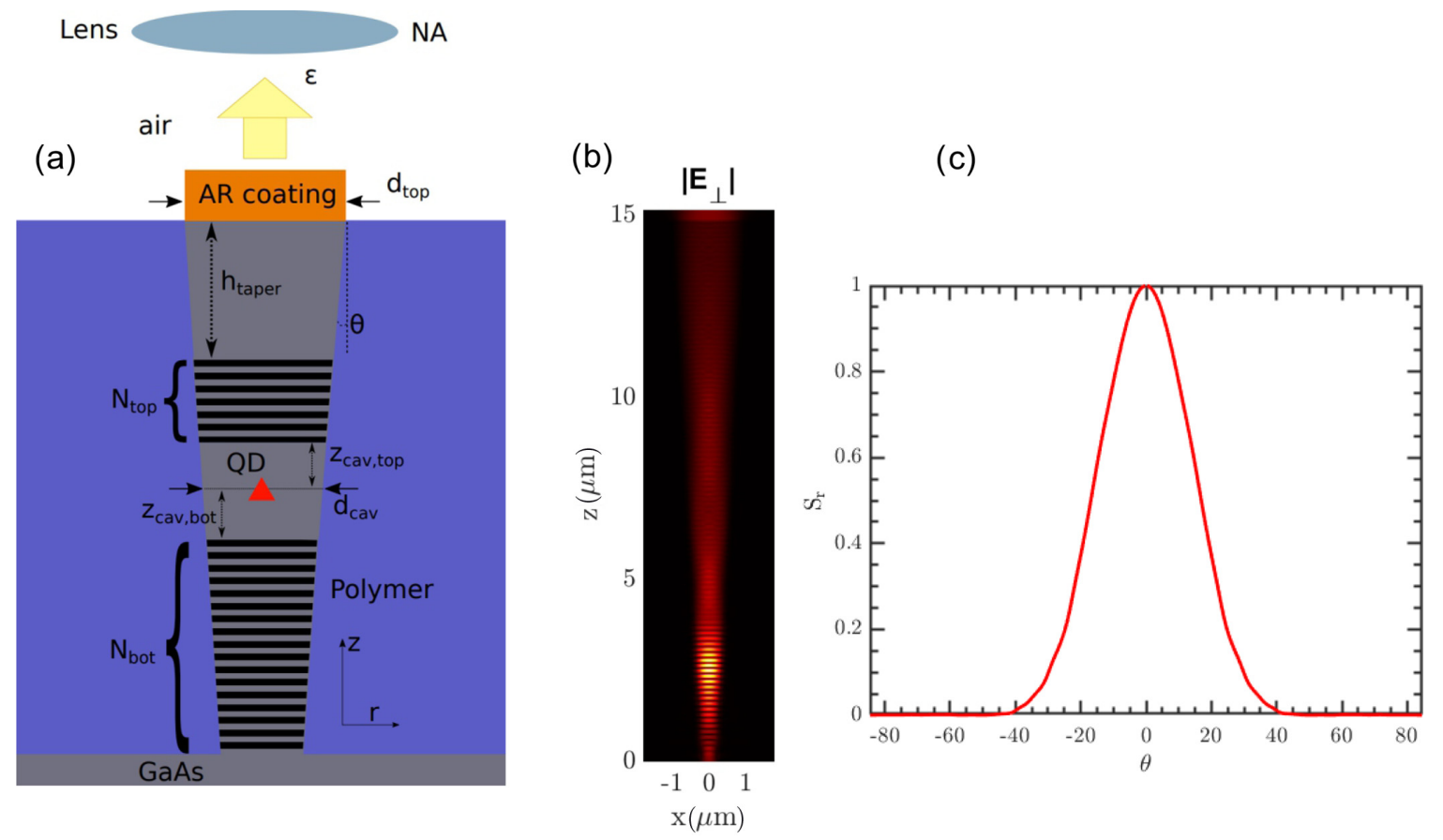

FIG. 1. Sketch of the simulated device. A quantum dot (QD) is centered in a $\lambda$ cavity $(\lambda=925 \mathrm{~nm})$ surrounded by DBR mirrors with $\mathrm{N}_{\text {bot }}=16\left(\mathrm{~N}_{\text {top }}=8\right)$ layer pairs in the bottom (top) mirror. The materials used are GaAs/ $\mathrm{Al}_{0.85} \mathrm{Ga}_{0.15} \mathrm{As}$. The structure has a constant sidewall angle of $\theta=3^{\circ}$. Above the top DBR, the structure features a taper of height $\mathrm{h}_{\text {taper }}$, and an AR coating. The refractive indices are: $\mathrm{n}_{\mathrm{GaAs}}=3.48$ [16], $\mathrm{n}_{\mathrm{AlGaAs}}=2.99$ [16], $\mathrm{n}_{\mathrm{AR}}=1.99$ [17], and $\mathrm{n}_{\text {clad }}=1.57$ [18]. (b) Electrical field profile of the cavity mode. (c) Normalized far-field emission pattern (radial component $\mathrm{S}_{r}$ of the Poynting vector).

of $\lambda_{\text {cav }}=925 \mathrm{~nm}$, the cavity (DBR layer) optical thickness is chosen as $\lambda / n_{\text {eff }}\left(\lambda / 4 n_{\text {eff }}\right)$ taking into account the diameterdependence of the effective refractive index $n_{\text {eff }}[13-15]$. To ensure optimal transmission to a Gaussian profile of a 0.8 numerical aperture $(\mathrm{NA}=0.8)$ lens, the top DBR is followed by a taper and an antireflective (AR) coating, where $h_{\text {taper }}$ is chosen as the smallest value ensuring that $d_{\text {top }} \geqslant 2 \mu \mathrm{m}$.

The simulations were performed using a Fourier modal method [19] with a true open geometry boundary condition [20] combined with a standard scattering matrix formalism [21]. This method allows direct access to individual eigenmodes and to cavity modes as discussed in detail in Ref. [15]. Furthermore, the QD is modeled as a classical dipole. The optical cavity mode profile is presented in Fig. 1(b), where we observe interference patterns in the DBR sections as also observed in micropillars as well as the adiabatic expansion of the fundamental mode in the top taper section. The corresponding far-field emission pattern shown in Fig. 1(c) features a Gaussian profile with low beam divergence. In Fig. 2(a) we show the $\mathrm{Q}$ factor of the cavity as a function of its diameter. For diameters below $2 \mu \mathrm{m}$ we observe fast oscillations before flattening to a constant value, which is similar to vertical micropillars [14,22,23]. Figure 2(b) shows the Purcell factor which reaches a value of approximately 3 for diameters in the range $500-625 \mathrm{~nm}$. As $F_{p}=\frac{3 Q \lambda_{\mathrm{cav}}{ }^{3}}{4 \pi^{2} n_{\mathrm{G} A s} V}$, where $V$ is the mode volume, the Purcell factor will decrease as we increase the diameter due to the increased mode volume. The $\beta$ factor, which describes the emission fraction into the cavity mode, is calculated as $\beta=\frac{P_{\text {cav }}}{P_{\text {total }}}$, where $P_{\text {total }}$ is the total emitted power and $P_{\text {cav }}$ is the power emitted into the cavity mode [15]. The $\beta$ factor is shown in Fig. 2(c). The $\beta$ factor follows the tendency of the Purcell factor and reaches a maximum value of $\beta=0.87$ at dcav $=500 \mathrm{~nm}$. The efficiency is defined as $\varepsilon=\frac{P_{\text {lens }}}{P_{\text {total }}}$, where $P_{\text {lens }}$ is the total collected power in a lens $\left(\mathrm{NA} \stackrel{I_{\text {total }}}{=} 0.8\right)$ taking into account an overlap with a Gaussian profile [15]. The efficiency is shown in Fig. 2(d) and follows the behavior of the $\beta$ factor and reaches a maximum value of $\varepsilon=0.73$ at $d_{\text {cav }}=725 \mathrm{~nm}$. The normalized power emission into the cavity mode is presented in terms of the generalized Purcell factor $F_{p}(\lambda)=P_{\text {cav }}(\lambda) / P_{0}(\lambda)$ [13], where $P_{0}$ is the power emitted in a bulk medium. The Purcell factor and the efficiency are presented in Figs. 2(e) and 2(f), respectively, as a function of wavelength, $\lambda$, at the optimal value of $d_{\text {cav }}=$ $725 \mathrm{~nm}$. The Purcell factor features a full width at half maximum of $4.4 \mathrm{~nm}$ corresponding to a $\mathrm{Q}$ of 211 . The efficiency spectrum features a similar behavior to $F_{p}$, where values of $\varepsilon \geqslant 0.7$ are obtained for wavelengths in the interval 924.8$927.1 \mathrm{~nm}$.

\section{DEVICE GROWTH}

The epitaxial structure for the devices was grown via molecular beam epitaxy on a 001 oriented GaAs wafer. The layer sequence has been optimized for a Bier-Glas shaped broadband photonic cavity. First, a 300-nm GaAs buffer was grown to smoothen the surface followed by 26 bottom DBR pairs of $\mathrm{Al}_{0.85} \mathrm{Ga}_{0.15} \mathrm{As}$ and by a $287-\mathrm{nm}$ thick GaAs cavity layer. The buffer layer is not shown in this scanning electron microscope (SEM) image. A detail of the cavity layer with the bottom and top mirror pair is shown in the inset of Fig. 3(a). The cavity layer contains $\operatorname{In}(\mathrm{G}$ (a)As QDs in the middle where the electric field forms an antinode. The second DBR consists 


$$
\text { (a) }
$$

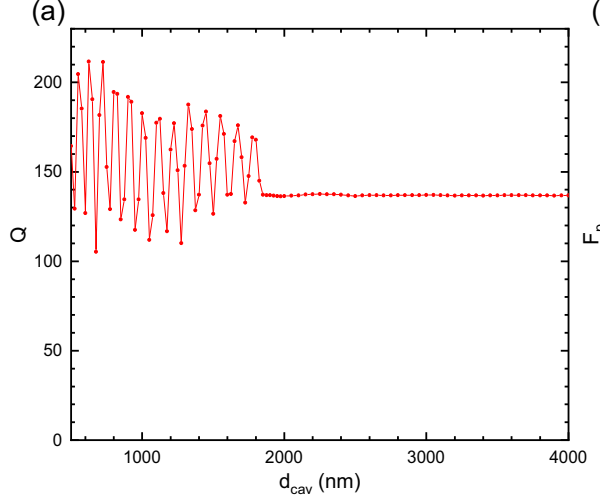

(b)
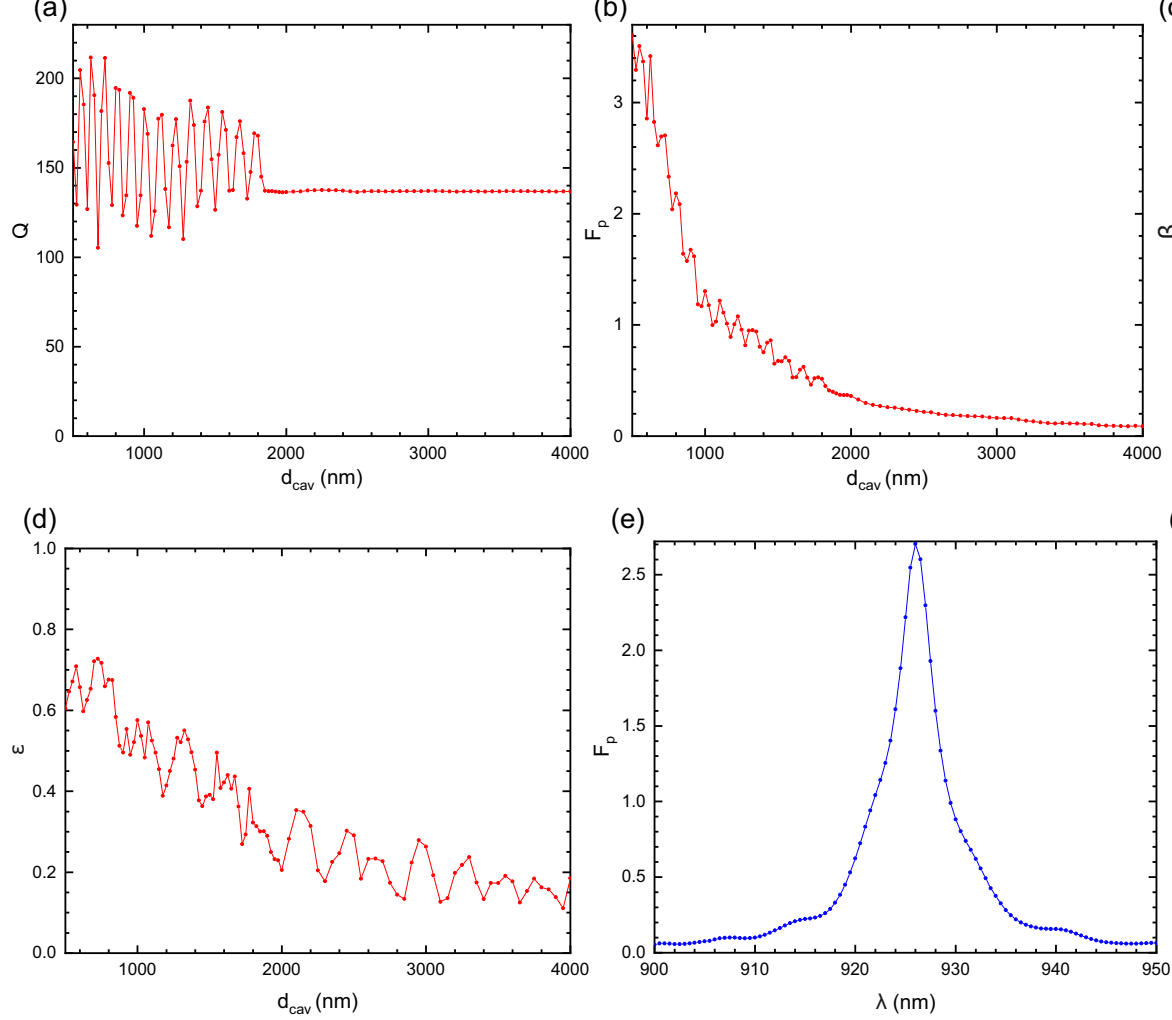

(e)

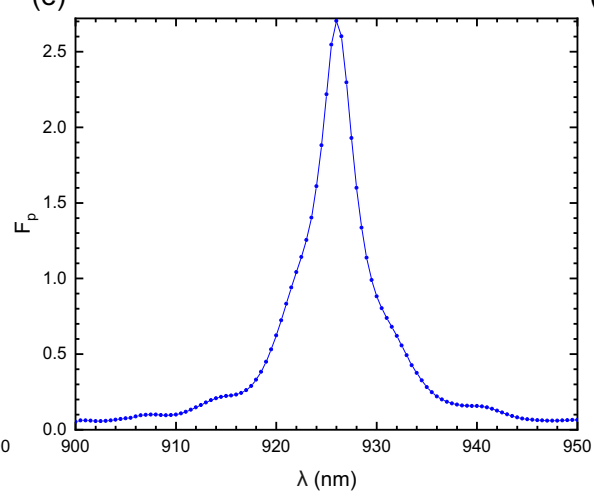

(c)

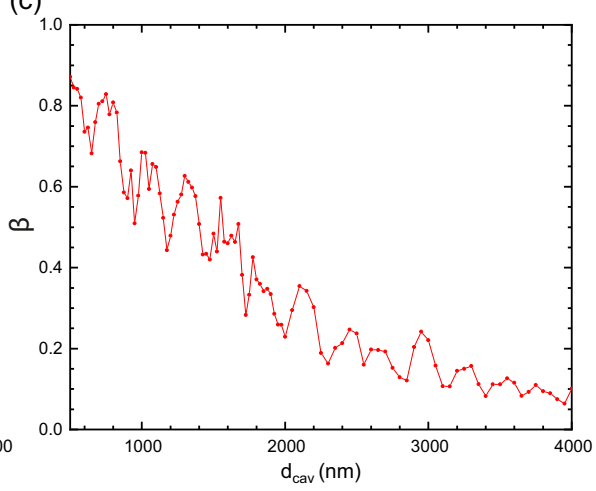

(f)

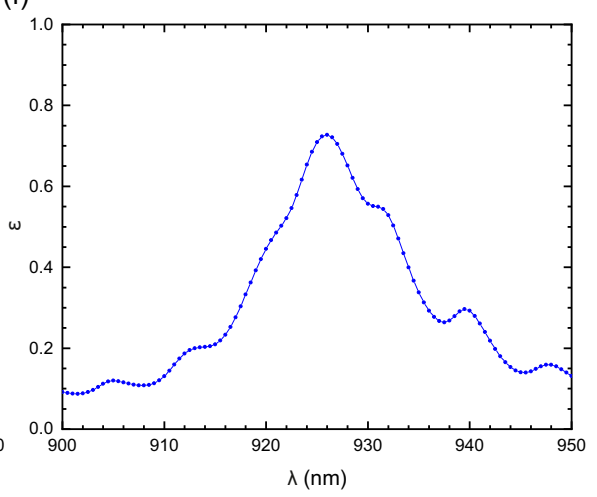

FIG. 2. (a) $\mathrm{Q}$ factor, (b) Purcell factor, (c) $\beta$ factor, and (d) efficiency, $\varepsilon$, as a function $d_{\text {cav. }}$. (e) Purcell factor and (f) efficiency as a function of wavelength, $\lambda$, at $d_{\text {cav }}=725 \mathrm{~nm}$. A 0.8 -NA lens is used.

of six mirror pairs. The structure is finished by a GaAs layer that is $10-\mu \mathrm{m}$ thick. The layer structure was designed to develop a stopband between 920 and $1020 \mathrm{~nm}$. The red curve in Fig. 3(b) depicts a transfer matrix simulation of the structure without the antireflective coating, while the black curve is the experimentally measured reflectivity spectrum of the planar structure. The oscillations in the stopband are caused by the $10 \mu \mathrm{m}$ GaAs layer, which is forming an additional Fabry-Pérot cavity, with the top DBR and the surface to air as its mirrors. A simulation of the structure without the thick
GaAs layer (blue curve in Fig. 3(b), shows the reflectivity of the DBR structure with the characteristic cavity resonance at $973 \mathrm{~nm}$.

\section{DEVICE FABRICATION}

To achieve the desired device shape, a systematic variation of the growth and the etching parameters was performed, as we describe in the following. The top diameters of the Bier-Glas structures were defined by electron beam (e-beam)
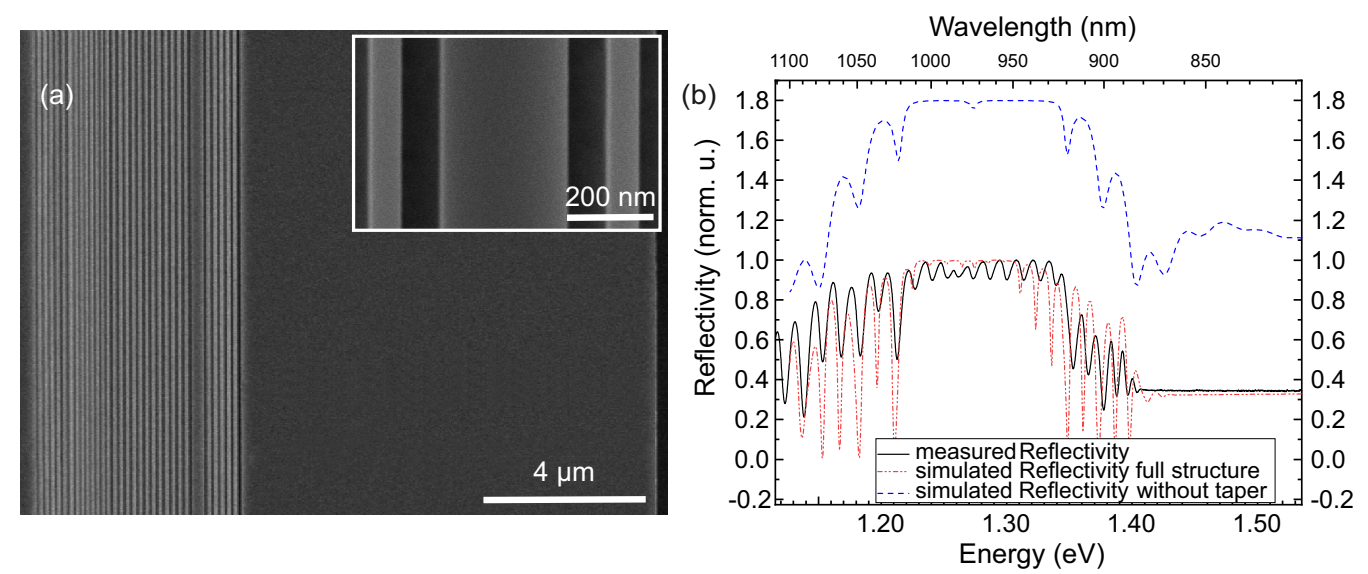

FIG. 3. (a) Cleaved edge SEM picture of the planar wafer. Two DBR sections (26 and 6 mirror pairs) separated by the cavity layer, which contains the InAs QDs, followed by $10 \mu \mathrm{m}$ GaAs (left to right). The cavity layer and the surrounding mirror-pairs can be seen in the inset. (b) Measured reflectivity of the planar structure (black) compared to the simulated reflectivity of the full structure (red) and the simulated structure without the $10-\mu \mathrm{m}$ GaAs top layer (blue with an offset of 0.8 ). 

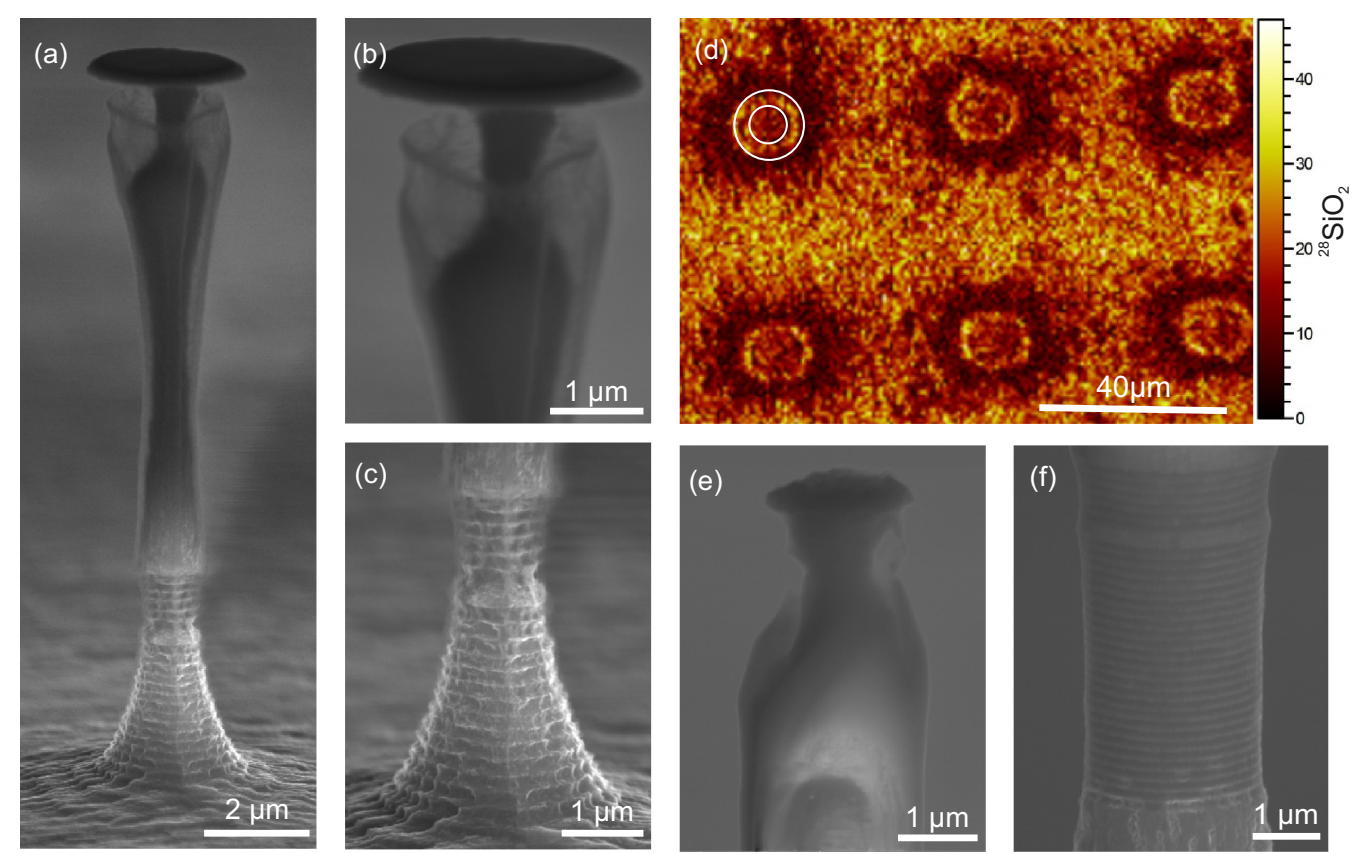

FIG. 4. SEM images of processed structures: (a) Etching the device on an undoped GaAs wafer yields an hourglass shape. Two unwanted process artefacts can be seen: first, a large under-etching beneath the $\mathrm{Cr} / \mathrm{BaF}$ mask and second the oxidation and significant roughening on the sidewalls [both detailed in (b)]. The oxidation is also visible in the DBR section of the structure [detailed in (c)]. Here, also an unwanted pronounced etching of the $\mathrm{Al}_{0.85} \mathrm{Ga}_{0.15}$ As layers (darker layers in the DBR segment) is clearly visible. (d) A static secondary-mass-spectroscopy measurement sensitive on [28] $\mathrm{SiO}_{2}$. The brighter areas around the pillars indicating an oxidation coating. For better visibility the area is highlighted in between the two white circles of the left upper pillar. (e) Images of a structure etched on a doped wafer. The shape is rather straight and has a constant angle within the whole device. The under etching in the surrounding of the mask is less than in the case of the undoped substrate. The DBR of this structure does not show selective etching and less sidewall oxidation [detailed in (f)] compared to the undoped wafer.

lithography, using a PMMA resist with Anisol as safer solvent. The diameter variation is in the range of $0.8-2.4 \mu \mathrm{m}$. Afterward, an etching mask of $\mathrm{Cr} / \mathrm{BaF}$ was evaporated on the surface. In the next step, the remaining photoresist was removed with a lift-off process and the pattern was transferred to the planar sample by using reactive electron cyclotron resonance plasma etching. Since the plasma etch process sensibly reacts on charge transport conditions in the sample, we first check the shape of nominally identical structures grown on a Si doped GaAs wafers as well as on undoped GaAs wafers with a SEM, shown in Fig. 4. Figures 4(a)-4(c) show a 14- $\mu \mathrm{m}$ high device, based on the heterostructure grown on an undoped GaAs wafer. The angle of the upper section (approx. $3^{\circ}$ ) is approximately ten times smaller than the angle in the lower DBR (approx. $25^{\circ}$ ) and increases towards the top of the taper section. The whole structure is covered with a transparent oxidation layer as also seen at micropillar structures before [24]. The thickness of this oxidation layer varies between $150 \mathrm{~nm}$ in the GaAs taper section and up to $280 \mathrm{~nm}$ in the DBR section. The composition of this oxidation layer was measured using a two-dimensional secondary-ionmass-spectroscopy technique. The scan shown in Fig. 4(d) displays a high density of [28] $\mathrm{SiO}_{2}$ next to the pillar center. For better visibility the area of interest is highlighted between the two white circles in the upper left pillar. The oxidation layer contains also other $\mathrm{SiO}_{2}$ isotopes. This layer has been attributed to a re-deposition effect stemming from remains of the $\mathrm{SiO}_{2}$ sample holder utilized in the etching chamber
[24-26], similar to the $\mathrm{SiO}_{2}$ mask used during the etching process of waveguides [27]. The structure is subject to a significant underetching beneath the etching mask [Fig. 4(b)]. The etching mask is visible as a black disk on top of the device (3- $\mu \mathrm{m}$ diameter). The remaining part of the GaAs right under the mask is $\sim 850$-nm thick and increases over a length of $\sim 2 \mu \mathrm{m}$ to a diameter of $1.5 \mu \mathrm{m}$. The behavior of the etching in the DBR segment also displays some peculiarities (Fig. 4(c): The DBR also shows strong selective oxidation and a rather rough surface with a pronounced modulation as seen before in etching processes [28]. This modulation stems from a different etching speed of the AlGaAs and GaAs layers. The etching as well as the oxidation of the $\mathrm{Al}_{0.85} \mathrm{Ga}_{0.15}$ As layers, is more pronounced due to the chemical etching and reactions of the residual etching products at the surface. The etch-anisotropy yields steps between the AlGaAs and GaAs layers as large as $100 \mathrm{~nm}$ and a step between the upper DBR and the GaAs taper. The waist of the structure has a width of $\sim 700 \mathrm{~nm}$ and is placed $\sim 500 \mathrm{~nm}$ vertically above the GaAs cavity layer. We note, that among multiple devices fabricated with this process, the minimal obtainable waist size was $500 \mathrm{~nm}$. Devices with smaller diameters systematically broke during the etching step due to mechanical stress, which most likely resulted from strong selective oxidation.

Utilizing the identical etching recipe on heterostructures grown on a $\mathrm{n}$-doped $(\mathrm{Si})$ wafer with a doping concentration between 1.7 and $3 \times 10^{18} \mathrm{~cm}^{-3}$ yields completely different results [Figs. 4(e) and 4(f)]. The structure shows a rather 


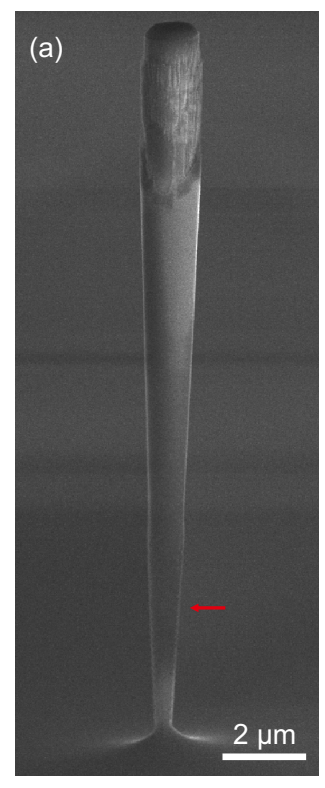

(e)

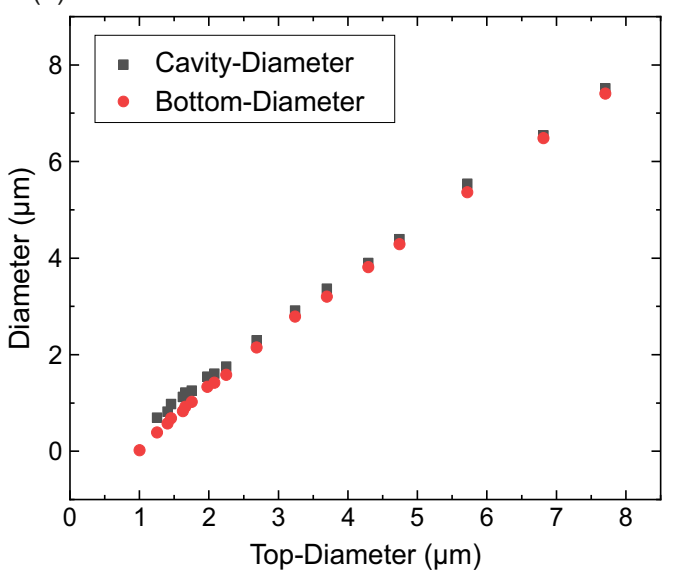

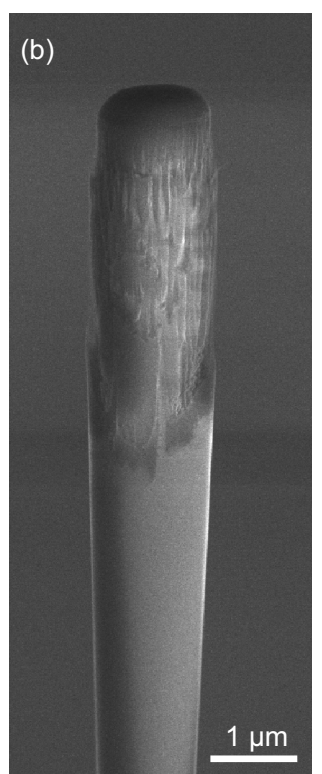
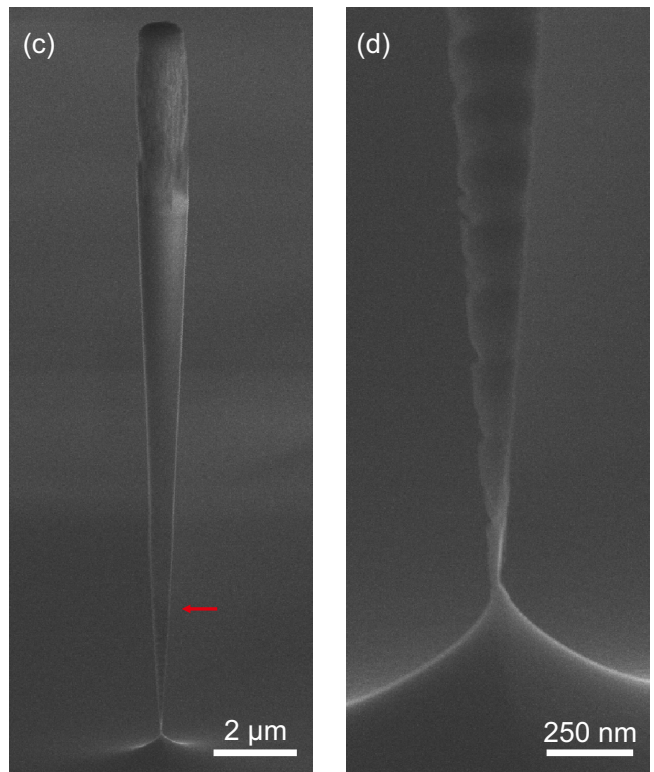

(f)

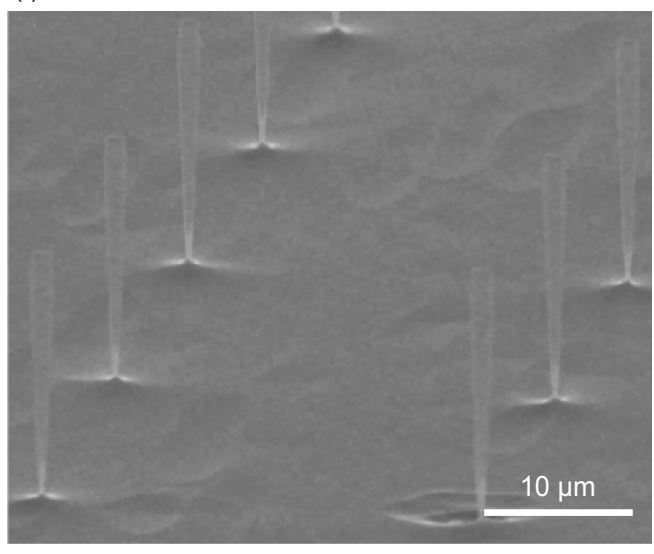

FIG. 5. SEM images of devices fabricated with optimized etching recipes. The position of the cavity layer is marked with an arrow. (a) A Bier-Glas structure with a top diameter of $1.25 \mu \mathrm{m}$ and a bottom diameter of $\sim 400 \mathrm{~nm}$. (b) detail of the top from (a) The top part of the device has a rougher sidewall in the vicinity of the $\mathrm{Cr} / \mathrm{BaF}$ etching mask, which is the black cover on the pillar top surface. (c) With this technique pillars with a top diameter of $1 \mu \mathrm{m}$ and a diameter as low as $\sim 20 \mathrm{~nm}$ at the thinnest part [detailed in (d)] can be achieved. (e) The diameter at the position of the cavity layer as well as at the narrowest position of the Bier-Glas at the transition to the foot is plotted as a function of the top device diameter. (f) An array of six standing Bier-Glases on a sample with the optimized etching recipe shows the high device yield. The position of the cavity layer is marked with a red arrow [Figs. 5(a) and 5(c)].

straight body with only a small angle between $1.6^{\circ}$ to $3^{\circ}$, which is constant from the top to the bottom of the device. Compared to the structure grown on an undoped substrate, the thickness of the coating on the sidewalls is significantly reduced $(\sim 50 \mathrm{~nm})$ and uniform over the whole device. The underetching at the region of the mask is still visible (Fig. 4) but not as pronounced as in the undoped wafer case.

Furthermore, the DBR section, detailed in Fig. 4(f), does not indicate selective etching of the AlGaAs and GaAs layers, except for a slightly highlighted cavity area. This result indicates a huge dependence of the etching behavior on different doping levels of the used wafer material. The choice of the wafer material strongly influences the shape and therefore the performance of the final device. An additional metal coating at the backside of the undoped wafer before the etching process leads to similar etching results like the doped wafers.

Since these results show very undesired features on undoped substrates, further device optimization was performed on n-doped substrates only. We optimized the previous etching recipe to get a more uniform sidewall angle and smaller diameters in the lower area of the device, as well as to reduce the persisting under etching beneath the $\mathrm{Cr} / \mathrm{BaF}$ etching mask. The optimization took place on an inductively coupled plasma (ICP) etching. We found the best results for an etching composition of 2:3 $\mathrm{Cl}_{2}, \mathrm{Ar}$, a high-frequency power of $50 \mathrm{~W}$ and an ICP power of $300 \mathrm{~W}$. The devices are compiled in Fig. 5. The devices on this sample have a variation in top diameter between 7.7 and $1 \mu \mathrm{m}$, allowing us to study the changes in the shape, as well as the differences in the optical properties 

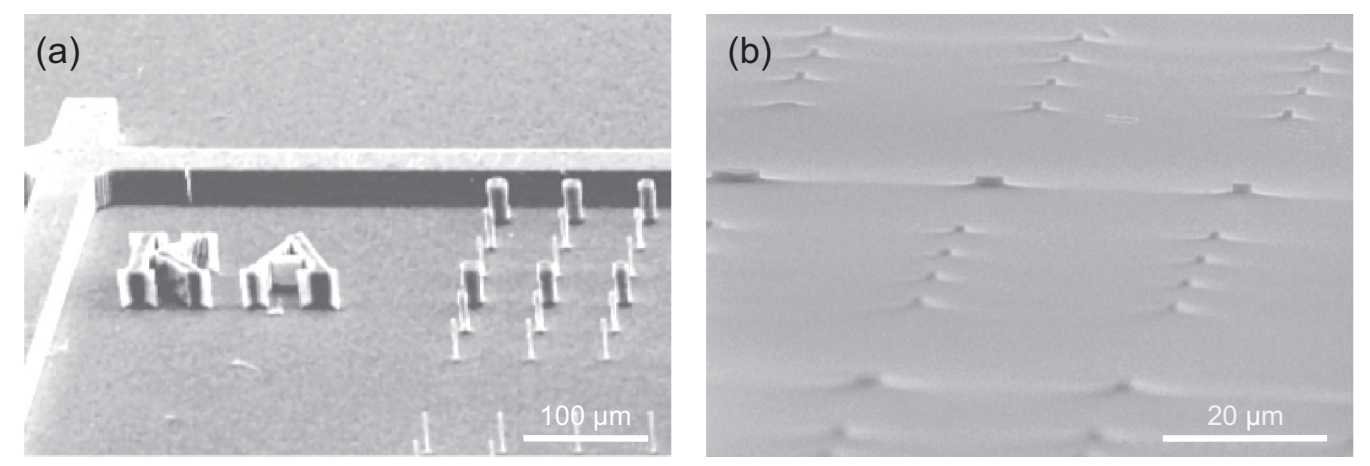

FIG. 6. (a) Global SEM image of the etched sample, including the protection wall to reduce the BCB flow. (b) SEM image of the successfully planarized sample.

of the devices. The device in Fig. 5(a) has a top diameter of $1.25 \mu \mathrm{m}$, a sidewall angle of $2^{\circ}$ around the cavity region which straightens towards the top, and a height of $\sim 15.9$ $\mu \mathrm{m}$. The shape of the device indeed resembles the canonical German Bier-Glas. The top of the Bier-Glas [Fig. 5(b)] still features rough sidewalls to a depth of $4 \mu \mathrm{m}$ under the etching mask. We believe that this results from charging of the device under the mask during the etching process, which causes a different etching result in this area. Another possibility involves the fact that the intrinsic oxide deposition from the sample holder is less efficient on the top of the device and causes a less pronounced sidewall cover.

All the device diameters (1-7.7 $\mu \mathrm{m}$ top diameter) have the same shape, examples of a $1.25-\mu \mathrm{m}$ diameter pillar and a 1- $\mu \mathrm{m}$ pillar can be seen in Figs. 5(a)-5(d), which leads to a linear dependence of (cavity and bottom) diameter to top-diameter [Fig. 5(e)]. The visibility of the Bier-Glas shape is mostly pronounced for small top-diameters because of the better contrast between taper and foot. A small deviation from the linear dependence is visible for top diameters smaller than $2.5 \mu \mathrm{m}$. Importantly, since the effects of etching selectivity between the GaAs and the AlGaAs mirrors and oxidation are strongly reduced with the optimized etching, the strain that builds up in the DBR segment of the device is dramatically reduced (compared to the devices which are presented in Figs. 4(a) and 4(b). Therefore, the device stability is significantly improved, allowing us to fabricate Bier-Glases with foot diameters as small as $20 \mathrm{~nm}$. Furthermore, for devices with foot diameters larger than $50 \mathrm{~nm}$, we see a quasi-100\% device yield, see Fig. 5(f). This is an extreme height to base relation in any dry etched GaAs-based cavity structure. The possibility to achieve those small diameters gives the chance for further design optimization in the future.

The $\mathrm{BaF} / \mathrm{Cr}$ hard mask is blocking light from the BierGlas, hindering its use as a photon source. Thus, a crucial step to conduct optical experiments is to remove the $\mathrm{BaF} / \mathrm{Cr}$ hard mask from the top. The hard mask is soluble in water, but this causes additional sidewall oxidation, especially in the aluminum containing layers of the DBR sections, and significantly damages the devices. A process that protects the sidewall while washing off the etch mask was developed in previous studies [29]. This process involves spin coating of liquid benzocyclobutene (BCB), which is subsequently hardened by a baking process. Afterwards the mask can be washed away in water. Unfortunately, the structures with thinner bottom diameters break during the spin coating, because of the critical aspect ratio of the Bier-Glas devices. To avoid spin coating, we drop BCB onto the sample and let it flow around the devices. To enhance the confinement of the liquid $\mathrm{BCB}$ protective walls were implemented within the etching step [see Fig. 6(a)] .The disadvantage is that the thickness of the $\mathrm{BCB}$ is not as uniform as with spin coating. In Fig. 6(b) one can see planarized Bier-Glases.

To finalize the fabrication process the devices are coated with a 126-nm- thick $\mathrm{Si}_{3} \mathrm{~N}_{4}$ AR layer on top of the sample [30]. To study the effect of the AR coating we investigated two samples. In Fig. 7(a) we show a microphotoluminescence $(\mu \mathrm{PL})$ spectrum of a device with a large top diameter of $7.7 \mu \mathrm{m}$ without antireflective coating. High excitation power was used to saturate all the single quantum dot transitions and to get access to the device mode structure. At $750-\mu \mathrm{W}$ excitation power we see that the modes start to redshift, which is an indication for local heating above $15 \mathrm{~K}$. We find at least six modes visible in the range from 935 to $985 \mathrm{~nm}$, with a mode spacing of $9 \pm 1 \mathrm{~nm}$. This spectrum is created by a Fabry-Pérot cavity formed between the surface to air and the upper DBR. The spectra of the exact same device with an antireflective coating is shown in Fig. 7(b). It is clearly visible that the AR coating suppresses the Fabry-Pérot modes, enabling us to capture the fundamental mode of the 7.7- $\mu \mathrm{m}$ Bier-Glas device, with a central wavelength of $965 \mathrm{~nm}$ and a resonance linewidth of $17.4 \pm 0.2 \mathrm{~nm}$.

\section{OPTICAL CHARACTERIZATION}

To characterize the optical performance of the devices, experiments in a $\mu \mathrm{PL}$ setup were performed. We excited the devices above band utilizing a green continuous wave (CW) laser with a wavelength of $532 \mathrm{~nm}$. The used objective has a NA of 0.42 and gives a focused spot size of $4.8 \pm 0.7 \mu \mathrm{m}$. The sample was mounted in a helium flow cryostat and cooled down to $10 \mathrm{~K}$.

Since the AR coating allows us to characterize the fundamental cavity mode, we study the dependence of the photonic confinement on its resonance energy [Fig. 7(a)]. The fundamental cavity mode of the Bier-Glas device shifts to higher energies with reduced diameter, resulting from the lateral photonic confinement [31]. While the QD density in our structures was sufficiently large $\left(3 \times 10^{9} \mathrm{~cm}^{-2}\right)$ to homogenously illuminate the cavity resonances for large diameters, the cavity 
(a) Wavelength $(\mathrm{nm})$

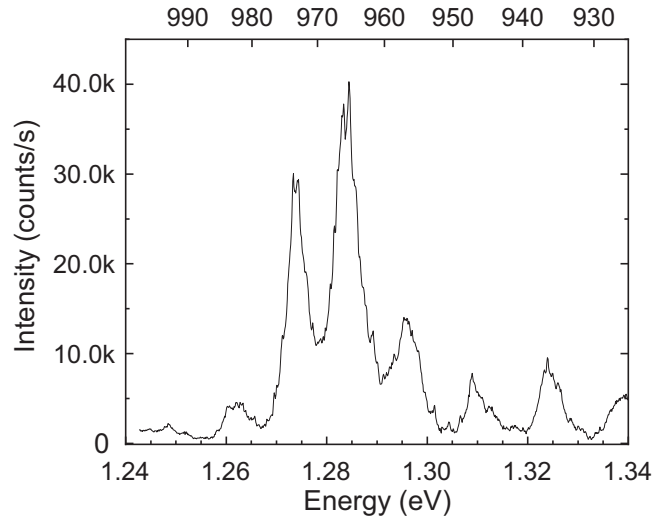

(b) Wavelength $(\mathrm{nm})$

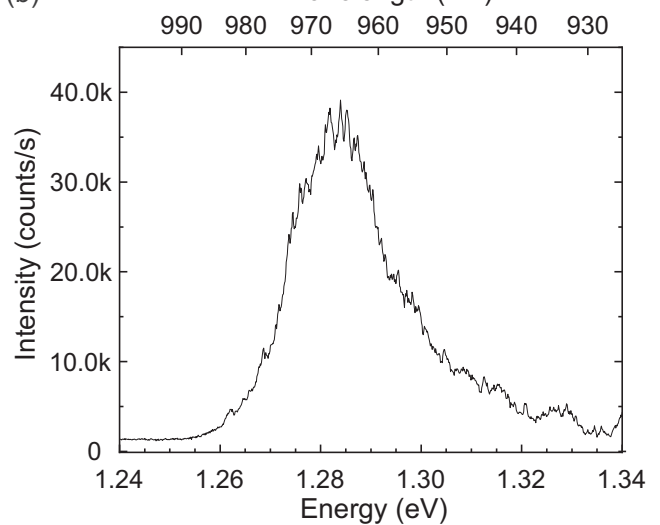

FIG. 7. Mode spectra of a Bier-Glas with a $7.7 \mu \mathrm{m}$ top diameter. (a) Spectra of the device without antireflective $\mathrm{Si}_{3} \mathrm{~N}_{4}$ coating and (b) with the antireflective coating. The fine spaced modes visible in (a) are formed by a Fabry-Pérot resonator which is consisting of the upper DBR and the GaAs to air interface. (b) $\mathrm{Si}_{3} \mathrm{~N}_{4}$ deposition recovers the underlying mode of the Bier-Glas device.

modes are strongly superimposed by single QD features in the smaller devices, adding some inaccuracy to the determination of the mode energies, which are plotted as a function of the cavity diameter in Fig. 7(b). The error bars are the standard deviation of various results from different pillars with the same size in combination with the uncertainties of the measurement itself. The variability of the ground mode energy is ranges between of $2.17 \mathrm{meV}$ for larger devices and $10 \mathrm{meV}$ of the smallest one.

While for the smallest devices, the experimental complications yield some increased fluctuations in the determined mode energies, the model, which is described above, nevertheless successfully reproduces the experimental shifts of the mode energies and supports our assignment of the broadband optical features to the fundamental cavity resonances.

To assess the performance of our structure as a quantum optical device, we studied single QD transitions in the device. The power-dependent emission of QDs in a device with a top diameter of $1.6 \mu \mathrm{m}$ can be seen in Fig. 8(a). We ramped the excitation power from $10 \mathrm{nW}$ up to $30 \mu \mathrm{W}$ where the QDs transitions are saturating. For further analysis, the two
QDs indicated by the black arrows are investigated by using a multipeak fit to extract the intensity of the emission lines. Furthermore, the line of interest is separated by at least $0.5 \mathrm{~nm}$ from other lines in the signal and can thus be resolved easily. The low energy QD at $943 \mathrm{~nm}$ is resonant with the fundamental cavity mode of the device, while the high energy QD (at $900 \mathrm{~nm}$ ) is spectrally far detuned from the cavity resonance. In Fig. 8(b) the normalized integrated intensity for the two QDs is plotted as a function of the excitation power. The saturation behavior of both emitters can be approximated via [32,33] $I=I_{\text {sat }}\left(1 /\left(1+P_{n} / P_{\text {exc }}\right)\right)$ with the excitation power $\mathrm{P}_{\text {exc }}$ and $\mathrm{P}_{n}$ as a fitting parameter to normalize the excitation power. The fits are the solid lines in Fig. 9(b). The saturation power and the saturation intensity of the QD in resonance with the cavity mode is used to normalize the excitation power and the integrated intensity. We note, that the saturation intensity of the resonant QD is almost 30 times larger than the saturation intensity of the off-resonant QD, as can be predicted in the model [Fig. 2(e)]. The measured intensity enhancement is a combination of Purcell effect and waveguide effects, which cannot be easily divided apart in our structure. As opposed (a)

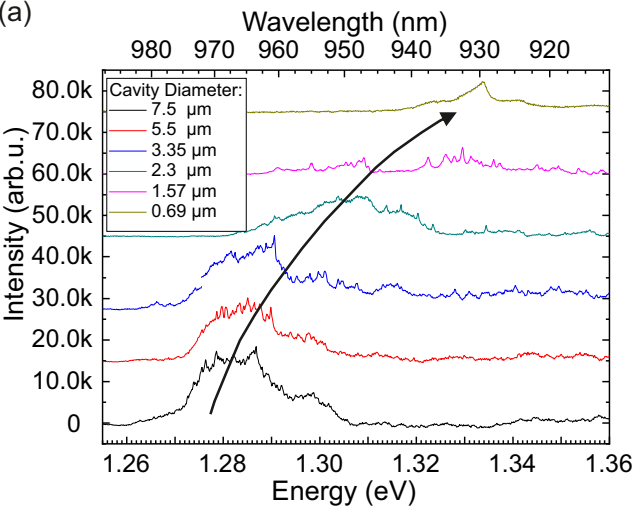

(b)

Diameter Cavity $(\mu \mathrm{m})$

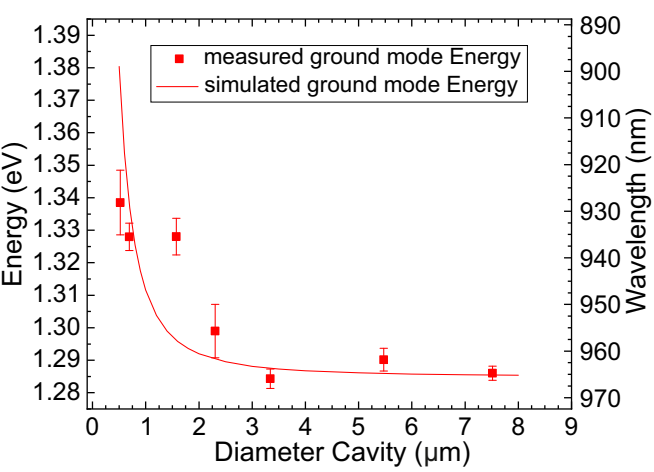

FIG. 8. (a) Cavity mode spectra for different Bier-Glas top diameters. A clear shift to higher energies is visible and highlighted by a black arrow. (b) Theoretical data for the ground mode energy (full orange line) and measured values for various diameters at the cavity waist. The error bars result from the standard deviation of various results from different pillars with the same size in combination with the uncertainties of the measurement itself. 

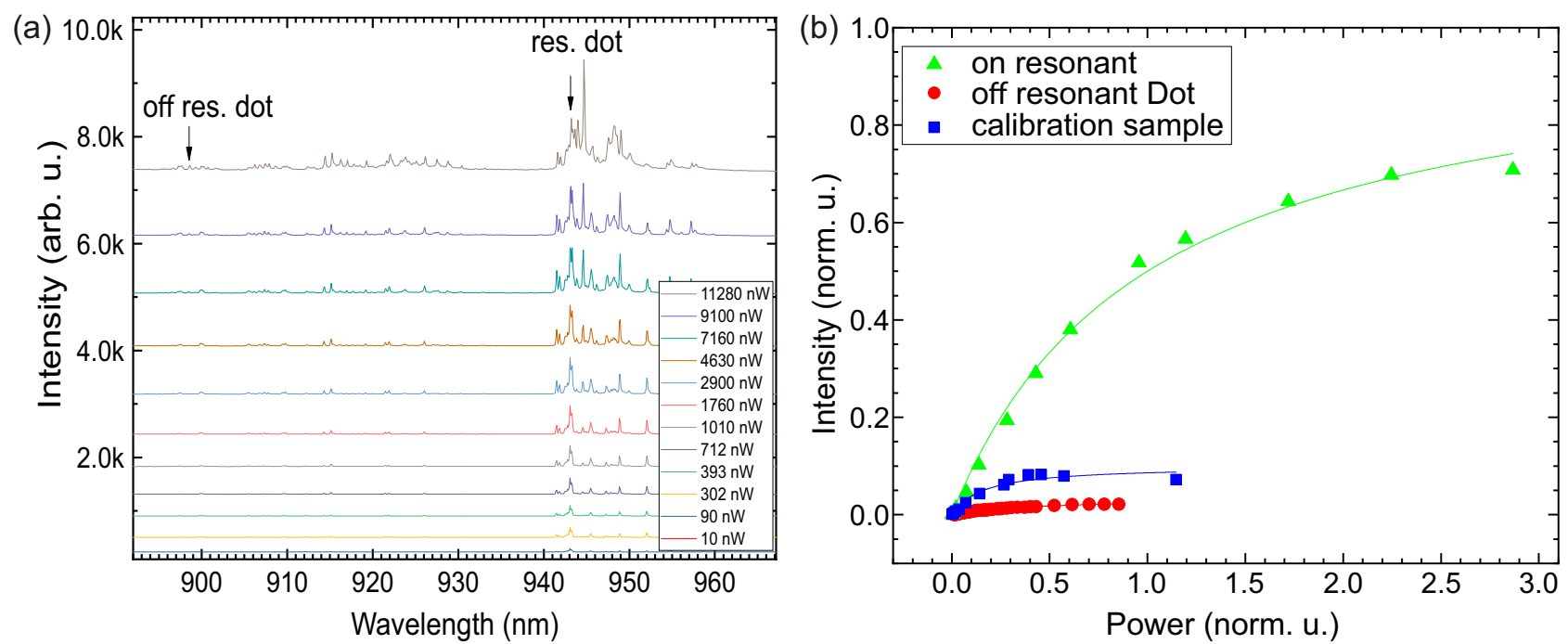

FIG. 9. (a) Intensity of a Bier-Glas device with a top diameter of $1.6 \mu \mathrm{m}$ for different excitation powers. Also indicated are two dotted lines, one resonant and the other one off resonant. The intensity (integrated under peak area) vs the excitation power for those two lines is compared in Fig. 8(b). The plots are fitted to extract the saturation power and intensity. The plot also contains a comparison of the Bier-Glas device with a planar QD calibration sample without cavity in the same way

to the common assumption for a QD in a conventional micropillar, where the Purcell can be extracted from the data of the Intensity of the off and on resonant QD [34], the offresonant QD is suppressed by the Bier-Glas structure, because in the waveguide the bulk mode density is modified. Thus, the Purcell factor cannot be directly extracted from the data, but we compare our intensity data to our theory, presented above. Since the measured data and the model agree in relative intensities, we assume the model to be correct and expect the Purcell factor to be close to the theory's upper bound of 2.7. The comparison of the Bier-Glas device to a QD reference sample with QDs in bulk and without a cavity is also shown in Fig. 8(b). Here, the QD resonant to the Bier-Glas structure reaches a saturation intensity ten times higher than the emitter in the reference structure, which underlines the impact of the Bier-Glas structure on the photon extraction.
Finally, we confirm the capability of our Bier-Glas cavities to act as nonclassical light sources by measuring their secondorder autocorrelation function on a device with a top diameter of 3.6 $\mu \mathrm{m}$. We excited the QD highlighted in Fig. 9(b), with a 532-nm CW laser at a power of $16 \mu \mathrm{W}$ and passed it to an autocorrelation setup. The coincidences show the expected antibunching behavior at zero-time delay [Fig. 9(a)]. The fitting of the data reveals $g_{\text {conv. }}^{(2)}(0)=0.366 \pm 0.10$. We assume that the value is nonzero, because of the finite time resolution of the detectors. To extract the correct value for $g^{2}(0)$, we convolve the expected $g^{(2)}$ function with a Gaussian distribution $G\left(t, t_{\text {res }}\right)$, with time resolution of the setup $t_{\text {res }}=260 \mathrm{ps}$. The resulting fitting formula is [35]

$$
g^{(2)}(\tau)=\left[1-\left(1-g_{\text {deconv. }}^{(2)}(0)\right) e^{\frac{-|\tau|}{\tau Q P}}\right] \otimes G\left(t, t_{\text {res }}\right)
$$
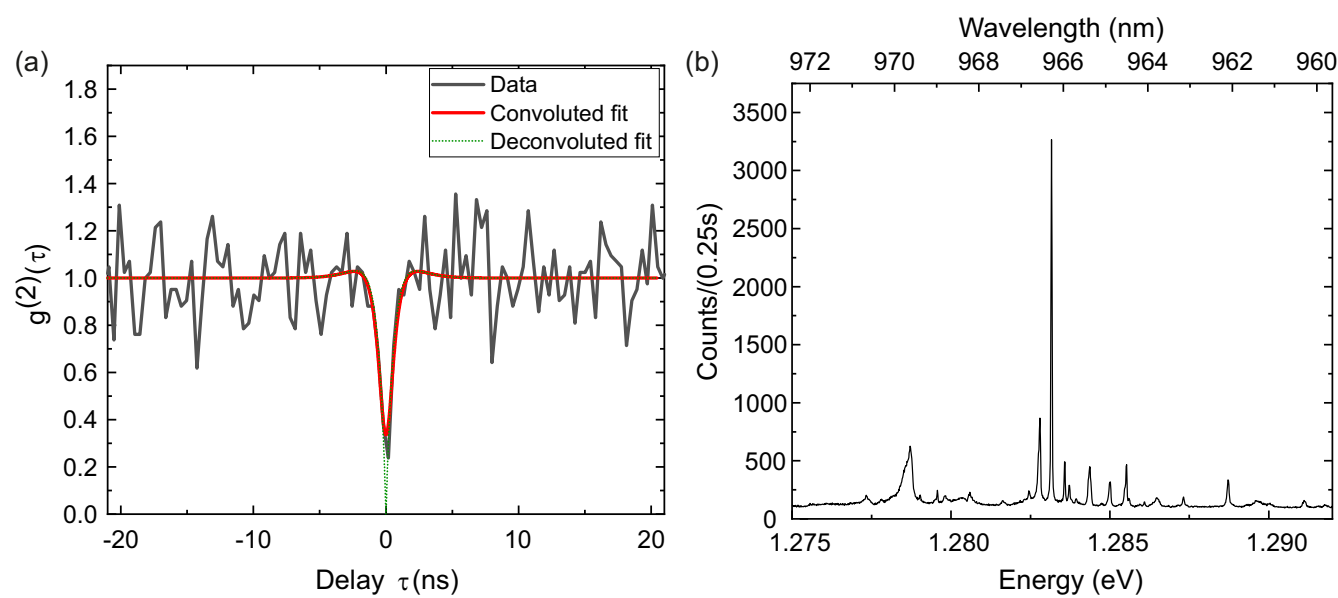

FIG. 10. (a) Coincidences vs the time delay is fitted with the second order correlation function. The convoluted fit gives a value of $g_{\text {conv. }}^{(2)}(0)=0.366 \pm 0.106$ for a time delay of $0 \mathrm{~ns}$. After deconvolution of the fit the value for zero delay is $g_{\text {deconv. }}^{(2)}(0)=0 \pm_{0.00}^{0.11}$. (b) The full PL spectrum of the Bier-Glas selected for the $g^{2}(\tau)$ measurement. 
[1] P. Michler, Quantum Dots for Quantum Information Technologies (Springer, Berlin, 2017).

[2] Bennett and DiVincenzo, Quantum information and computation, Nature (London) 404, 247 (2000).

[3] H.-J. Briegel, W. Dür, J. I. Cirac, and P. Zoller, Quantum Repeaters: The Role of Imperfect Local Operations in Quantum Communication, Phys. Rev. Lett. 81, 5932 (1998).

[4] S. Unsleber, Y.-M. He, S. Gerhardt, S. Maier, C.-Y. Lu, J.-W. Pan, N. Gregersen, M. Kamp, C. Schneider, and S. Höfling, Highly indistinguishable on-demand resonance fluorescence photons from a deterministic quantum dot micropillar device with 74\% extraction efficiency, Opt. Express OE 24, 8539 (2016).

[5] Xing Ding, Yu He, Z.-C. Duan, Niels Gregersen, M.-C. Chen, S. Unsleber, S. Maier, Christian Schneider, Martin Kamp, Sven Höfling, Chao-Yang Lu, and Jian-Wei Pan, On-Demand Single Photons with High Extraction Efficiency and Near-Unity Indistinguishability from a Resonantly Driven Quantum Dot in a Micropillar, Phys. Rev. Lett. 116, 020401 (2016).

[6] N. Somaschi, V. Giesz, L. De Santis, J. C. Loredo, M. P. Almeida, G. Hornecker, S. L. Portalupi, T. Grange, C. Antón, J. Demory, C. Gómez, I. Sagnes, N. D. Lanzillotti-Kimura, A. Lemaítre, A. Auffeves, A. G. White, L. Lanco, and P. Senellart, Near-optimal single-photon sources in the solid state, Nat. Photonics 10, 340 (2016).

[7] S. Unsleber, D. P. S. McCutcheon, M. Dambach, M. Lermer, N. Gregersen, S. Höfling, J. Mørk, C. Schneider, and M. Kamp, Two-photon interference from a quantum dot microcavity: Persistent pure dephasing and suppression of time jitter, Phys. Rev. B 91, 075413 (2015).

[8] C. Santori, D. Fattal, J. Vucković, G. S. Solomon, and Y. Yamamoto, Indistinguishable photons from a single-photon device, Nature (London) 419, 594 (2002).

[9] T. Grange, N. Somaschi, C. Antón, L. De Santis, G. Coppola, V. Giesz, A. Lemaître, I. Sagnes, A. Auffèves, and P. Senellart, Reducing PhononInduced Decoherence in Solid-State Single- broadband efficiencies with substantial Purcell enhancement. Furthermore, our technological advancement, allowing us to produce DBR-based cavities supported by $20-\mathrm{nm}$ feet certainly can pave the way towards quantum-optomechanic applications [36] by increasing the force sensibility of possible structures [37].

\section{ACKNOWLEDGMENTS}

We gratefully acknowledge financial support from the state of Bavaria. The project HYPER-U-P-S has received funding from the QuantERA ERA-NET Co-fund in Quantum Technologies implemented within the European Union's Horizon 2020 Programme. M.A.J. and N.G. acknowledge support from the European Research Council (ERC-CoG "UNITY", Grant No. 865230) and from the Independent Research Fund Denmark (Grant No. DFF-9041-00046B). We acknowledge Technical assistance by A. Wolf and M. Emmerling, as well as support by Dr. C. Anton-Solanas during the characterization of the devices.
Photon Sources with Cavity Quantum Electrodynamics, Phys. Rev. Lett. 118, 253602 (2017).

[10] J. Claudon, J. Bleuse, N. S. Malik, M. Bazin, P. Jaffrennou, N. Gregersen, C. Sauvan, P. Lalanne, and J.-M. Gérard, A highly efficient single-photon source based on a quantum dot in a photonic nanowire, Nature (London) Photon 4, 174 (2010).

[11] M. A. M. Versteegh, M. E. Reimer, K. D. Jöns, D. Dalacu, P. J. Poole, A. Gulinatti, A. Giudice, and V. Zwiller, Observation of strongly entangled photon pairs from a nanowire quantum dot, Nat. Commun. 5, 5298 (2014).

[12] T. Huber, A. Predojević, M. Khoshnegar, D. Dalacu, P. J. Poole, H. Majedi, and G. Weihs, Polarization entangled photons from quantum dots embedded in nanowires, Nano Lett. 14, 7107 (2014).

[13] N. Gregersen, D. P. S. McCutcheon, J. Mørk, J.-M. Gérard, and J. Claudon, A broadband tapered nanocavity for efficient nonclassical light emission, Opt. Express 24, 20904 (2016).

[14] P. Lalanne, J.-P. Hugonin, and J.-M. Gérard, Electromagnetic study of the quality factor of pillar microcavities in the small diameter limit, Appl. Phys. Lett. 84, 4726 (2004).

[15] B.-Y. Wang, E. V. Denning, U. M. Gür, C.-Y. Lu, and N. Gregersen, Micropillar single-photon source design for simultaneous near-unity efficiency and indistinguishability, Phys. Rev. B 102, 125301 (2020).

[16] S. Gehrsitz, F. K. Reinhart, C. Gourgon, N. Herres, A. Vonlanthen, and H. Sigg, The refractive index of $\mathrm{AlxGa}_{1-x}$ As below the band gap: Accurate determination and empirical modeling, J. Appl. Phys. 87, 7825 (2000).

[17] T. Bååk, Silicon oxynitride: a material for GRIN optics, Appl. Opt. 21, 1069 (1982)

[18] T. Hanemann, J. Böhm, K. Honnef, E. Ritzhaupt-Kleissl, and J. Haußelt, Polymer/phenanthrene-derivative host-guest systems: Rheological, optical and thermal properties, Macromol. Mater. Eng. 292, 285 (2007).

[19] U. M. Gür, S. Arslanagić, M. Mattes, and N. Gregersen, Open-geometry modal method based on transverse electric and 
transverse magnetic mode expansion for orthogonal curvilinear coordinates, Phys. Rev. E 103, 033301 (2021).

[20] T. Häyrynen, J. R. de Lasson, and N. Gregersen, Opengeometry Fourier modal method: Modeling nanophotonic structures in infinite domains, J. Opt. Soc. Am. A 33, 1298 (2016).

[21] A. V. Lavrinenko, Numerical Methods in Photonics (CRC Press, Boca Raton, FL, 2015).

[22] N. Gregersen, S. Reitzenstein, C. Kistner, M. Strauss, C. Schneider, S. Hofling, L. Worschech, A. Forchel, T. R. Nielsen, J. Mork, and J.-M. Gerard, Numerical and experimental study of the Q factor of high-Q micropillar cavities, IEEE J. Quantum Electron. 46, 1470 (2010).

[23] N. Gregersen, P. Kaer, and J. Mørk, Modeling and design of high-efficiency single-photon sources, IEEE J. Sel. Top. Quantum Electron. 19, 1 (2013).

[24] S. Reitzenstein, N. Gregersen, C. Kistner, M. Strauss, C. Schneider, L. Pan, T. R. Nielsen, S. Höfling, J. Mørk, and A. Forchel, Oscillatory variations in the $\mathrm{Q}$ factors of high quality micropillar cavities, Appl. Phys. Lett. 94, 61108 (2009).

[25] G. Lecamp, J. P. Hugonin, P. Lalanne, R. Braive, S. Varoutsis, S. Laurent, A. Lemaître, I. Sagnes, G. Patriarche, I. RobertPhilip, and I. Abram, Submicron-diameter semiconductor pillar microcavities with very high quality factors, Appl. Phys. Lett. 90, 91120 (2007).

[26] S. Varoutsis, S. Laurent, I. Sagnes, A. Lemaître, L. Ferlazzo, C. Mériadec, G. Patriarche, I. Robert-Philip, and I. Abram, Reactive-ion etching of high-Q and submicron-diameter GaAs/AlAs micropillar cavities, J. Vac. Sci. Technol. B 23, 2499 (2005).

[27] M. Volatier, D. Duchesne, R. Morandotti, R. Arès, and V. Aimez, Extremely high aspect ratio GaAs and GaAs/AlGaAs nanowaveguides fabricated using chlorine ICP etching with N2promoted passivation, Nanotechnology 21, 134014 (2010).

[28] G. S. Solomon, M. Pelton, and Y. Yamamoto, Single-Mode Spontaneous Emission from a Single Quantum Dot in a ThreeDimensional Microcavity, Phys. Rev. Lett. 86, 3903 (2001).
[29] C. Böckler, S. Reitzenstein, C. Kistner, R. Debusmann, A. Löffler, T. Kida, S. Höfling, A. Forchel, L. Grenouillet, J. Claudon, and J. M. Gérard, Electrically driven high-Q quantum dot-micropillar cavities, Appl. Phys. Lett. 92, 91107 (2008).

[30] A. D. Osterkryger, J. Claudon, J.-M. Gérard, and N. Gregersen, Photonic "hourglass", design for efficient quantum light emission, Opt. Lett. 44, 2617 (2019).

[31] T. Gutbrod, M. Bayer, A. Forchel, J. P. Reithmaier, T. L. Reinecke, S. Rudin, and P. A. Knipp, Weak and strong coupling of photons and excitons in photonic dots, Phys. Rev. B 57, 9950 (1998).

[32] S. Kumar, A. Kaczmarczyk, and B. D. Gerardot, Strain-induced spatial and spectral isolation of quantum emitters in mono- and bilayer $\mathrm{WSe}_{2}$, Nano Lett. 15, 7567 (2015).

[33] L. N. Tripathi, O. Iff, S. Betzold, Ł. Dusanowski, M. Emmerling, K. Moon, Y. J. Lee, S.-H. Kwon, S. Höfling, and C. Schneider, Spontaneous emission enhancement in straininduced $\mathrm{WSe}_{2}$ monolayer-based quantum light sources on metallic surfaces, ACS Photon. 5, 1919 (2018).

[34] M. Munsch, A. Mosset, A. Auffèves, S. Seidelin, J. P. Poizat, J.-M. Gérard, A. Lemaître, I. Sagnes, and P. Senellart, Continuous-wave versus time-resolved measurements of Purcell factors for quantum dots in semiconductor microcavities, Phys. Rev. B 80, 115312 (2009).

[35] P. Michler, A. Imamoğlu, M. D. Mason, P. J. Carson, G. F. Strouse, and S. K. Buratto, Quantum correlation among photons from a single quantum dot at room temperature, Nature (London) 406, 968 (2000).

[36] M. Munsch, A. V. Kuhlmann, D. Cadeddu, J.-M. Gérard, J. Claudon, M. Poggio, and R. J. Warburton, Resonant driving of a single photon emitter embedded in a mechanical oscillator, Nat. Commun. 8, 76 (2017).

[37] P. Paulitschke, N. Seltner, A. Lebedev, H. Lorenz, and E. M. Weig, Size-independent Young's modulus of inverted conical GaAs nanowire resonators, Appl. Phys. Lett. 103, 261901 (2013). 